\title{
Diseño y validación de una escala de uso de medios académicos virtuales durante la pandemia COVID-19
}

\section{Design and validation of a scale of use of virtual academia during the COVID-19 pandemic}

\author{
Oscar Mamani- Benito* \\ Universidad Peruana Unión, Juliaca, Perú \\ ORCID-ID: https://orcid.org/0000-0002-9818-2601 \\ Manuel Landa-Barzola \\ Universidad Cesar Vallejo, Lima, Perú \\ Asociación Peruana de Profesionales de las Adicciones, Perú \\ ORCID: https://orcid.org/0000-0002-7090-8845 \\ Renzo Felipe Carranza Esteban \\ Universidad San Ignacio de Loyola, Lima, Perú \\ ORCID: https://orcid.org/0000-0003-4086-4845 \\ Ana Elguera-Pajares \\ Universidad San Ignacio de Loyola, Lima, Perú \\ ORCID: https://orcid.org/0000-0003-2163-6502

\section{Christian R. Mejia} \\ Universidad Continental, Huancayo, Perú \\ ORCID-ID: https://orcid.org/ 0000-0002-5940-7281
}

Recibido 20-01-21 Revisado 23-04-21 Aceptado 28-06-21 En línea 06-09-21

*Correspondencia

Email: oscar.mb@upeu.edu.pe
Citar como:

Mamani, O., Landa, M., Carranza, R., Elguera, A., \& Mejia, C. (2021). Diseño y validación de una escala de uso de medios académicos virtuales durante la pandemia COVID-19. $\begin{array}{llll}\text { Propósitos } y \quad \text { Representaciones, } & 9(2), \quad 1390 .\end{array}$ http://dx.doi.org/10.20511/pyr2021.v9n2.1390 


\section{Resumen}

Es necesario evaluar los entornos de las clases virtuales que se han generado a causa de la pandemia COVID-19, ante ello, el objetivo fue diseñar y validar una escala de uso de medios académicos virtuales. El estudio es de tipo instrumental, donde la muestra estuvo conformada por 582 universitarios peruanos. La escala estuvo conformada por 10 ítems distribuidos en 2 dimensiones. Para hallar la validez de contenido se consideró el juicio de expertos, para la validez de constructo el análisis factorial exploratorio y confirmatorio, y para estimar la confiabilidad el $\alpha$ de Cronbach. Los resultados indican que los ítems recibieron una evaluación favorable (V de Aiken $>.70)$. Los índices de bondad de ajuste fueron satisfactorios $\left(\chi^{2}=24.204, d f=13 ; p=.001 ; \mathrm{RMR}=.058\right.$; CFI $=.931 ; \mathrm{TLI}=.908 ; \mathrm{GFI}=.957 ; \mathrm{AGFI}=.931$ y RMSEA $=.071)$, asimismo, la correlación entre el factor 1 y 2 fue significativa $(\mathrm{p}<0.05)$ y la confiabilidad buena $(\alpha=.74$; IC 95\% $=.70-.77)$. Se concluye que la escala de uso de medios académicos virtuales durante la pandemia COVID-19 es una herramienta breve y cuenta con evidencia de validez y confiabilidad.

Palabras clave: Medios virtuales, COVID-19, Educación a distancia, Perú

\section{Summary}

It is necessary to evaluate the virtual class environments that have been generated by the COVID-19 pandemic. In view of this, the objective was to design and validate a scale for the use of virtual academic media. The study is of an instrumental type, where the sample was conformed by 582 Peruvian university students. The scale was conformed by 10 items distributed in 2 dimensions. In order to find the validity of the content, it was considered the experts' judgment, for the validity of the construct, the exploratory and confirmatory factorial analysis, and to estimate the reliability, the Cronbach's $\alpha$ The results indicate that the items received a favorable evaluation (Aiken's $\mathrm{V}>.70$ ). Goodness-of-fit indices were satisfactory $(\chi 2=24,204$, df $=13 ; \mathrm{p}=.001$; RMR $=.058$; $\mathrm{IFC}=.931 ; \mathrm{TLI}=.908 ; \mathrm{GFI}=.957 ; \mathrm{AGFI}=.931$ and $\mathrm{RMSEA}=.071)$, and the correlation between factor 1 and 2 was significant $(\mathrm{p}<0.05)$ and reliability good $(\alpha=.74 ; 95 \% \mathrm{CI}=$ .70 - .77). It is concluded that the COVID-19 pandemic virtual academia scale is a short tool and has evidence of validity and reliability.

Keywords: Virtual Media, COVID-19, Distance Education, Perú.

\section{Introducción}

El surgimiento de un nuevo brote de coronavirus ha generado preocupación en la comunidad científica y la sociedad en general (Palacios et al., 2020). Esta enfermedad, denominada COVID-19, es una infección viral altamente transmisible y patógena que tiene su causa en el coronavirus 2 del síndrome respiratorio agudo severo (SARS-CoV-2, por sus siglas en inglés) (Shereen et al., 2020).

El impacto que ha ocasionado no solo ha afectado a la salud pública, sino, también se han visto repercusiones en la economía, el ámbito laboral (Chakraborty \& Maity, 2020) y, especialmente la educación. Respecto a este último, muchos países han optado por el 
cierre temporal de los centros enseñanza (Viner et al., 2020). A nivel superior, las universidades han tenido que recurrir a tecnologías educativas e implementar plataformas virtuales para desarrollar las clases mientras se cumple el aislamiento social impuesto por los gobiernos de turno (Aquino-Canchari \& Medina-Quispe, 2020; João \& CarvalhoFilho, 2020). No obstante, no demoraron en aparecer algunas limitantes, sobre todo de adecuación a las tecnologías educativas por parte de los docentes y, de adaptación al nuevo proceso de enseñanza aprendizaje por parte de los universitarios (Ángel et al., 2017).

La puesta en marcha de entornos virtuales ha sido objeto de estudio desde hace muchos años; por ejemplo, una investigación (Inzunza et al., 2012) halló que los alumnos de ciencias básicas se mostraron dispuestos a trabajar en ambientes mixtos de aprendizaje donde se involucran clases presenciales apoyadas por entornos virtuales. Datos como estos hacen pensar que son los jóvenes quienes parecen tener mayor aceptación por la enseñanza virtual, sin embargo, es necesario reconocer que es todo un desafío gestionar su aprendizaje ligado a las expectativas y necesidades por satisfacer (Juca, 2016).

En contextos como el actual, a causa de la COVID-19, los estudiantes se enfrentan a un contexto de aprendizaje en solitario, donde necesitarán ciertas habilidades para adaptarse a los entornos virtuales (Orozco \& García, 2017), ya que su capacidad de autoconocimiento y autorregulación determinan su rendimiento académico (Córdova et al., 2019). Esta situación lo convierte en el principal protagonista de su aprendizaje, donde el profesor no es más que un mediador de este proceso (Moreira-Segura \& DelgadilloEspinoza, 2015).

En base a lo mencionado por Fernández y Rivero (2014) se puede entender que el uso de medios académicos virtuales implica el hecho de recurrir a aquellos entornos y herramientas digitales como complemento para la gestión académica y administrativa del proceso de enseñanza-aprendizaje. Un fenómeno que según la literatura científica ha sido foco de interés para los investigadores, quienes propusieron su medición a través de instrumentos documentales; así, tenemos el caso de Deshwal et al. (2017) quienes validaron una escala de experiencia de aprendizaje en línea y su impacto en la satisfacción de alumnos de la India; de igual modo, Berridi et al. (2015) validaron una escala de interacción en contextos virtuales de aprendizaje para estudiantes universitarios en México; también, Carvalho y De Oliveira (2016) construyeron y validaron una escala de estrategias de enseñanza, aprendizaje y motivación de aprendizaje en línea (EEAMAVA) para estudiantes brasileros. Por último, Moneta et al. (2017) adaptaron y validaron un instrumento para medir la interacción en b-learning en estudiantes argentinos.

En el Perú, se ha encontrado algunos estudios, aunque no necesariamente de corte instrumental; por ejemplo, Copari (2014) analizó la influencia de la enseñanza virtual sobre el aprendizaje en estudiantes de Puno. En Lima, Castro-Rodríguez y LaraVerástegui (2018) evaluaron la percepción de estudiantes de posgrado sobre la implementación del enfoque b-learning y, en Chiclayo Saavedra-Idrogo y SaavedraParedes (2017) evaluaron si la aplicación de un programa metodológico basado en el uso del entorno virtual Moodle mejoraba los aprendizajes en el curso de cirugía. Por lo visto, no se tiene reportes de investigaciones que hayan validado o generado instrumentos para evaluar el uso de medios académicos virtuales en el contexto universitario (Britez, 2020), sobre todo, para el contexto de la pandemia COVID-19.

Es por lo mencionado, que el presente estudio tiene el objetivo de diseñar y validar una escala de uso de medios académicos virtuales durante la pandemia COVID-19 para población universitaria peruana. 


\section{Método}

\section{Participantes}

A través de un muestreo no probabilístico intencional, se tuvo la participación de 582 estudiantes universitarios peruanos, 339 fueron mujeres $(58,2 \%)$, cuyas edades oscilaban entre 16 y 41 años (Media $=21,8$ años y DS $=5,1$ ). Se excluyeron a los que no completaron el cuestionario (la exclusión fue menor de 5\%).

\section{Diseño del instrumento}

Se realizó una revisión bibliográfica en las bases de datos SciELO y Scopus, a fin de construir el marco teórico y definir conceptualmente el constructo. Se siguió la propuesta teórica de Fernández y Rivero (2014) y se diseñó la escala de Uso de medios académicos virtuales ante la pandemia COVID-19 (EUMAV-Cov19). La escala, está compuesta por 10 ítems (se seleccionaron en base a la relevancia y coyuntura actual de la emergencia sanitaria en el contexto académico universitario), distribuidos en 2 dimensiones, cuyas opciones de respuesta son totalmente en desacuerdo, en desacuerdo, ni de acuerdo ni en desacuerdo, de acuerdo y totalmente de acuerdo.

\section{Procedimientos}

El estudio tuvo fases. Primera, la EUMAV-Cov19 fue diseñada, analizada y revisada por el equipo de investigación. Segunda, se analizó la evidencia de la validez de contendido, a fin de analizar la relevancia, representatividad y claridad de los ítems teniendo como grupo de expertos a ocho ingenieros especialistas en TICs y dos psicólogos especialistas en psicometría; todos ejercían la docencia universitaria. Tercera, se realizó un focus group compuesto por 36 jueces experienciales (estudiantes universitarios), con el propósito de identificar si hubiera, dificultad para comprender la claridad de los ítems o preguntas ambiguas. Cuarta, se recolectó la información a través de un formulario de Google, que comenzó con una hoja de consentimiento informado, seguido de un apartado que indicó los fines del estudio y donde se enfatizó que la participación era voluntaria, anónima y confidencial.

\section{Análisis de datos}

En primer lugar, se analizó la evidencia de validez basada en el contenido a través del juicio de expertos y de acuerdo a los puntajes asignados a los ítems se calculó el coeficiente $\mathrm{V}$ de Aiken (con valores significativos $\geq .70$ ) y sus intervalos de confianza (IC) al 95\% (Ventura-León, 2019). En segundo lugar, se calculó la media, desviación estándar, asimetría y curtosis (estadísticos descriptivos). En tercer lugar, se realizó el análisis factorial exploratorio (AFE) previa aplicación del test de Bartlett y el coeficiente de Kaiser-Meyer-Olkin (KMO), en base a una matriz de correlaciones policóricas. Se consideró el método por mínimos cuadrados no ponderados con rotación oblicua promin y para la determinación del número de factores se utilizó el análisis paralelo. En cuarto lugar, se realizó un análisis factorial confirmatorio (AFC), considerando índices de bondad de ajuste: Chi-cuadrado ( $\chi 2)$, índice de Tucker-Lewis (TLI,), índice de bondad de ajuste (GFI), índice ajustado de bondad de ajuste (AGFI), el índice de ajuste incremental (CFI), la confirmación del modelo hipotético, se darán por medio de un ajuste aceptable (> .900) o un ajuste optimo (> .950). Por otro lado, el índice de error de cuadrático medio 
(RMR) y error cuadrático medio de aproximación (RMSEA), valores aceptables lo más cercanos a cero $(<.070)$. Finalmente se calculó la fiabilidad de la escala.

Para calcular los estadísticos descriptivos y el AFE se utilizó el programa FACTOR Analysis 10.1, para el AFC el software AMOS versión 24.0 y para calcular la fiabilidad, el software estadístico SPSS versión 24.0.

\section{Resultados}

Para analizar la relevancia, representatividad y claridad de los ítems de la Escala de uso de medios virtuales durante la pandemia COVID-19, se solicitó el juicio de diez expertos, los puntajes asignados se cuantificaron con el coeficiente V de Aiken. Se aprecia en la Tabla 1 que todos los ítems recibieron una evaluación favorable por parte de los expertos $(\mathrm{V}>.70)$. En cuanto a la relevancia, se encontró que los ítems 1 y 3 son los más importantes ( $\mathrm{V}=1$; IC 95\%: .89 - 1), los ítems 1, 3, 4, 5, y 9 son los más representativos (V = .93; IC 95\%: .79 - .98). Respecto a la claridad, el ítem 2 fue mejor evaluado (V = .97; IC 95\%: .83 - .99). Asimismo, se aprecia que todos los valores del límite inferior (Li) del IC 95\% son apropiados y todos los valores del coeficiente V fueron estadísticamente significativos.

Tabla 1.

$V$ de Aiken para la evaluación de la relevancia, representatividad y claridad de los ítems de la EUMAV-Cov19.

\begin{tabular}{|c|c|c|c|c|c|c|c|c|c|c|c|c|}
\hline \multirow{2}{*}{ Ítems } & \multicolumn{4}{|c|}{ Relevancia $(n=10)$} & \multicolumn{4}{|c|}{$\begin{array}{c}\text { Representatividad }(\mathrm{n}= \\
10)\end{array}$} & \multicolumn{4}{|c|}{ Claridad $(\mathrm{n}=10)$} \\
\hline & $\mathrm{M}$ & $\begin{array}{l}\mathrm{D} \\
\mathrm{E}\end{array}$ & V & $\%$ & M & $\begin{array}{l}\mathrm{D} \\
\mathrm{E}\end{array}$ & V & IC & $\mathrm{M}$ & $\begin{array}{l}\mathrm{D} \\
\mathrm{E}\end{array}$ & V & $5 \%$ \\
\hline Ítem 1 & 3 & .00 & 1 & $89-1$ & 2.8 & .42 & .93 & $.79-$ & 2.8 & .42 & .93 & $.79-.98$ \\
\hline ítem 2 & 2.9 & .32 & .97 & $.83-.99$ & 2.7 & .48 & .90 & .74 & 2.9 & .32 & .97 & .99 \\
\hline ítem 3 & 3 & .00 & 1 & $89-1$ & 2.8 & .42 & .93 & $.79-.98$ & 2.8 & .42 & .93 & .98 \\
\hline ítem 4 & 2.7 & .48 & .90 & $.74-.97$ & 2.8 & .42 & .93 & $.79-.98$ & 2.8 & .42 & .93 & $.79-.98$ \\
\hline ítem 5 & 2.7 & .48 & .90 & $.74-.97$ & 2.8 & .42 & .93 & $.79-.98$ & 2.7 & .48 & .90 & . $74-.97$ \\
\hline ítem 6 & 2.5 & .71 & .83 & $.66-.93$ & 2.4 & .70 & .80 & $.63-.90$ & 2.6 & .52 & .87 & . $70-.95$ \\
\hline ítem 7 & 2.7 & .48 & .90 & $.74-.97$ & 2.6 & .52 & .87 & $.70-.95$ & 2.6 & .52 & .87 & . $70-.95$ \\
\hline ítem 8 & 2.9 & .32 & .97 & $.83-.99$ & 2.6 & .52 & .87 & $.70-.95$ & 2.5 & .53 & .83 & $.66-.93$ \\
\hline ítem 9 & 2.9 & .32 & .97 & $.83-.99$ & 2.8 & .42 & .93 & $.79-.98$ & 2.8 & .42 & .93 & $.79-.98$ \\
\hline $\begin{array}{c}\text { ítem } \\
10\end{array}$ & 2.8 & .42 & .93 & $.79-.98$ & 2.7 & .48 & .90 & 97 & 2.7 & .48 & .90 & $.74-.97$ \\
\hline
\end{tabular}

Nota: $\mathrm{M}=$ media; $\mathrm{DE}$ = desviación estándar; $\mathrm{V}$ = coeficiente V de Aiken; IC 95\% = Intervalo de confianza de la V de Aiken.

\section{Análisis preliminar de los ítems}

La Tabla 2 muestra los estadísticos descriptivos de los diez ítems de la EUMAV-Cov19. Se aprecia que el ítem 3 tiene la mayor media $(\mathrm{M}=4.12)$ y el ítem 10 la más baja $(\mathrm{M}=$ 2.95). Respecto a la variabilidad, el ítem $8(\mathrm{DE}=1.17)$ muestra la mayor dispersión. Asimismo, se observa que los ítems 3 y 6 de la escala presentan valores elevados de asimetría y curtosis $(> \pm 1.5)$. 
Tabla 2

Análisis preliminar de los ítems de la escala EUMAV-Cov19.

\begin{tabular}{lcccc}
\hline Ítem & $\mathrm{M}$ & $\mathrm{DE}$ & $\mathrm{As}$ & $\mathrm{K}$ \\
\hline Ítem 1 & 3.844 & .966 & -.853 & .574 \\
Ítem 2 & 3.900 & .901 & -.961 & .888 \\
Ítem 3 & 4.119 & .858 & -1.373 & 2.529 \\
Ítem 4 & 2.940 & 1.143 & .056 & -.847 \\
Ítem 5 & 3.782 & .956 & -.867 & .591 \\
Ítem 6 & 4.017 & .829 & -1.138 & 2.008 \\
Ítem 7 & 3.378 & 1.104 & -.473 & -.579 \\
Ítem 8 & 3.024 & 1.171 & -.085 & -.879 \\
Ítem 9 & 3.070 & 1.049 & -.150 & -.518 \\
Ítem 10 & 2.947 & 1.046 & .007 & -.702 \\
\hline
\end{tabular}

Nota $: \mathrm{M}=$ Media; $\mathrm{DE}=$ Desviación estándar; As = Coeficiente de asimetría; $\mathrm{K}$ = Coeficiente de curtosis.

\section{Análisis Factorial Exploratorio (AFE)}

Se realizó un AFE previo cálculo del índice KMO (.800) y la prueba de Bartlett (2087.1; $\mathrm{gl}=45 ; \mathrm{p}<.001)$, los índices fueron aceptables. Se utilizó el método de mínimos cuadrados no ponderados con rotación oblicua promin, además, para la determinación de factores se utilizó el análisis paralelo, indicando la existencia de 2 factores. La solución rotada de los diez ítems explica el 55.27\% de la varianza total explicada. El factor 1 (Uso de medios virtuales) explica $37.65 \%$ de la varianza y se conforma con los ítems $1,2,3$, 5,6 y 7, con saturaciones mayores a 40 y el Factor 2 (Apoyo en el aprendizaje) aporta el $17.62 \%$ de la varianza y está conformado por los ítems $4,8,9$ y 10, con saturaciones mayores a .42 .

Tabla 3

Análisis factorial exploratorio de la escala EUMAV-Cov19

\begin{tabular}{lll}
\hline \multicolumn{1}{c}{ Ítems } & F1 & F2 \\
\hline $\begin{array}{l}\text { 1. Considero necesario el uso de plataformas o sistemas virtuales para } \\
\text { estudiar. }\end{array}$ & .613 \\
$\begin{array}{l}\text { 2. Uso las plataformas y/o sistemas virtuales de mi universidad para } \\
\text { gestionar mis cursos. }\end{array}$ & .751 \\
$\begin{array}{l}\text { 3. Uso herramientas tecnológicas como Zoom, Google-Meet u otras } \\
\text { para recibir las clases virtuales. }\end{array}$ & .832 \\
$\begin{array}{l}\text { 5. Mi universidad pone a disposición recursos académicos (repositorio y y } \\
\text { biblioteca digital) para mi aprendizaje. }\end{array}$ & .555 \\
$\begin{array}{l}\text { 6. Uso motores de búsqueda y bases de datos como Google, Yahoo, etc. } \\
\text { / Scielo, Redalyc, Scopus, WoS, para cumplir con mis tareas. }\end{array}$ & .670 \\
$\begin{array}{l}\text { 7. Uso gestores de información como Mendeley, Zotero, EndNote, } \\
\text { Citavi u otros, para mis trabajos de investigación y/o tareas. }\end{array}$ & .417 \\
$\begin{array}{l}\text { 4. Me cuesta adaptarme al uso de las plataformas virtuales para mis } \\
\text { clases. }\end{array}$ & &
\end{tabular}




\begin{tabular}{|c|c|c|}
\hline $\begin{array}{l}\text { 8. Usar herramientas virtuales como Zoom, Google-Meet u otras, no } \\
\text { ayudan a mi aprendizaje. }\end{array}$ & & .466 \\
\hline $\begin{array}{l}\text { 9. Las clases virtuales no favorecen mi aprendizaje ni mi rendimiento } \\
\text { académico. }\end{array}$ & & .892 \\
\hline $\begin{array}{l}\text { 10. Las evaluaciones virtuales no garantizan } \mathrm{mi} \text { aprendizaje ni mi } \\
\text { rendimiento académico. }\end{array}$ & & .786 \\
\hline$\%$ de varianza & $\begin{array}{c}37.6 \\
5\end{array}$ & 17.62 \\
\hline \multicolumn{3}{|l|}{ Correlación inter-factores } \\
\hline F1 & 1 & \\
\hline $\mathrm{F} 2$ & $\begin{array}{c}.359 \\
*\end{array}$ & 1 \\
\hline
\end{tabular}

Nota: F1: Uso de medios virtuales; F2: Apoyo en el aprendizaje.

\section{Análisis factorial confirmatorio}

En la Tabla 4 se aprecia el AFC, el cual se utilizó para verificar la evidencia de validez; basada en la estructura interna de la EUMAV COVID-19 (diez ítems distribuidos en 2 factores). Los índices de bondad de ajuste fueron satisfactorios $\left(\chi^{2}=24.204, d f=13 ; p=\right.$ $.001 ; \mathrm{RMR}=.058 ; \mathrm{CFI}=.931 ; \mathrm{TLI}=.908 ; \mathrm{GFI}=.957 ; \mathrm{AGFI}=.931$ y RMSEA $=.071)$, confirmando así el modelo original (Figura 1). Asimismo, las correlaciones entre el factor 1 y 2 fueron significativas $(\mathrm{p}<.05)$.

Tabla 4

Índices de ajuste de los modelos factoriales de la escala EUMAV-Cov19

\begin{tabular}{|c|c|c|c|c|c|c|c|c|c|c|}
\hline Modelo & $\chi^{2}$ & $\begin{array}{l}\mathrm{g} \\
1\end{array}$ & $\mathrm{p}$ & CFI & TLI & GFI & $\begin{array}{c}\text { AGF } \\
\text { I }\end{array}$ & RMSEA & CMIN/DF & $\begin{array}{c}\mathrm{RM} \\
\mathrm{R}\end{array}$ \\
\hline $\begin{array}{l}\text { Origina } \\
1\end{array}$ & $\begin{array}{c}134.43 \\
1\end{array}$ & $\begin{array}{l}3 \\
4\end{array}$ & $<.001$ & .931 & .908 & .957 & .931 & .071 & 3.954 & .058 \\
\hline
\end{tabular}

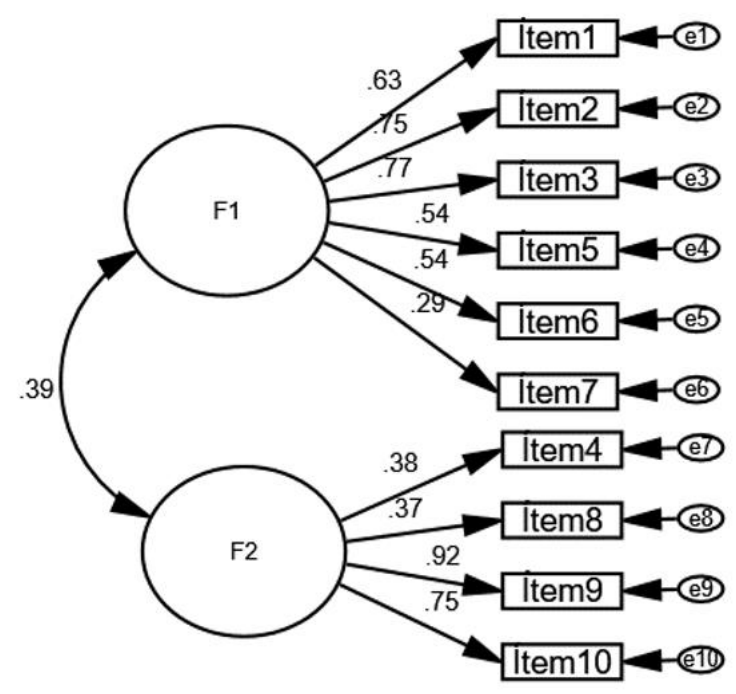

Figura 1. Modelo de la escala EUMAV-Cov19 


\section{Confiabilidad}

Para evidenciar la estabilidad de las puntuaciones de la prueba, se empleó el método de consistencia interna, que fue calculada a través del coeficiente Alfa, obteniendo valores aceptables $(>.70)$ (Taber, 2018). En la escala general $(\alpha=.74$; IC 95\% = .70 - .77), asimismo, para el factor $1(\alpha=.74$; IC 95\% $=.70-.77)$ y en el factor $2(\alpha=.70$; IC 95\% $=.65-.73)$; evidenciando que las puntuaciones de la escala son confiables y consistentes entre sí.

\section{Discusión}

Se diseñó y validó una escala para evaluar el uso de medios académicos virtuales durante la pandemia COVID-19, para el contexto universitario. Debido a que se ha optado por brindar educación de manera remota en todos los niveles educativos, incluido la superior.

La UNESCO (2020) elaboró un listado donde las instituciones y/o el estudiante puede hacer uso de las diferentes plataformas virtuales. Ello ofrece la oportunidad de construir un nuevo instrumento que mida el uso de esos medios virtuales.

La evidencia de estructura interna proporcionada mediante el Análisis Factorial Exploratorio sustenta que el análisis paralelo sugirió la existencia de dos factores y con cargas factoriales aceptables, debido a que los 10 ítems obtuvieron valores adecuados (Lloret-Segura et al., 2014). El modelo a priori de dos factores, mediante el Análisis Factorial Confirmatorio demostró obtener bondades de ajustes aceptables; asimismo, la carga interfactorial obtuvo un valor adecuado. Es importante destacar, que la EUMAVCov19 se fundamentó en análisis lógicos y empíricos, el cual demuestra el grado en que las relaciones de los ítems y sus factores se ajustan al constructo investigado (American Educational Research Association, 2018).

El modelo propuesto de dos factores muestra congruencia entre sí, dado que, el entorno de aprendizaje virtual es captado de manera profunda cuando el estudiante tiene el interés por conocer las diferentes plataformas, ello conllevaría a que se adapte adecuadamente al nuevo aprendizaje adquirido (Jelfs \& Colbourn, 2002). Por el contrario, los estudiantes que desarrollan un enfoque superficial se quejan de la falta de apoyo, ya que, no llegan a completar ninguna de las tareas, debido al desconocimiento del uso de las plataformas virtuales (Mimirinis \& Bhattacharya, 2007). En concordancia, con la teoría de Fernández y Rivero (2014), los adecuados entornos virtuales de enseñanza, contribuyen significativamente en el estudiante, ya que genera un nivel de motivación más alto, interés por el aprendizaje y por el uso de medios virtuales; de esta manera, el entorno de aprendizaje virtual favorece al desarrollo del individuo.

Con respecto a las evidencias de confiabilidad, que fue calculada mediante el método de consistencia interna y calculada a través del coeficiente alfa, la escala total y las dimensiones demostraron valores adecuados. Estos resultados son esperados, debido a que la escala contiene un formato breve, el cual puede ser usado para investigación (sin embargo, esto no aplicaría del todo para entornos clínicos), donde el valor estándar vendría a ser $>.90$ (Prieto \& Delgado, 2010). Los resultados adquiridos por esta construcción son las primeras evidencias psicométricas de la Escala de Uso de Medios Académicos Virtuales (EUMAV-Cov19). Se plantea la replicación de más revisiones psicométricas en cuanto a la confiabilidad de las puntuaciones de la escala. 
La investigación contiene algunas limitaciones. En primer lugar, la metodología y la recolección de datos se llevó a cabo solo una vez (consistencia interna), sería importante poder contar con revisiones que examinen la estabilidad de las puntuaciones de los ítems en el tiempo, proceso que se desarrolla mediante el método test-retest (Prieto \& Delgado, 2010). En segundo lugar, no se realizó la evidencia de validez con otras variables, su posterior revisión podría relacionar examinar el grado de predictibilidad de la variable con construcciones relacionadas o diferentes, para poder proporcionar una fuente importante de evidencias de validez (American Educational Research Association, 2018).

Se concluye que la Escala de uso de medios académicos virtuales (EUMAVCov19) es una versión breve y que, debido a sus adecuadas propiedades psicométricas en evidencias basadas en el contenido, estructura interna y confiabilidad; puede ser de uso exclusivo para otros diseños de investigación en educación a distancia y otras áreas afines, dado que la medición de los test psicómetros son importantes para todo tipo de investigaciones (Furr, 2011).

\section{Referencias}

American Educational Research Association. (2018). Estándares para Pruebas Educativas y Psicológicas. https://www.jstor.org/stable/j.ctvr43hg2

Ángel, C., Váldes, J., \& Guzmán, T. (2017). Límites, desafíos y oportunidades para enseñar en los mundos virtuales. Innovación Educativa, 17(75), 149-168. https://www.redalyc.org/pdf/1794/179454112008.pdf

Aquino-Canchari, C., \& Medina-Quispe, C. (2020). COVID-19 y la educación en estudiantes de medicina. Revista Cubana de Investigaciones Biomedicas, 39(2), 2 5. http://www.revibiomedica.sld.cu/index.php/ibi/article/view/758/pdf

Ato, M., López, J., \& Benavente, A. (2013). Un sistema de clasificación de los diseños de investigación en psicología. Anales de Psicología, 29(3), 1038-1059. http://scielo.isciii.es/scielo.php?script=sci_arttext\&pid=S021297282013000300043

Berridi, R., Martínez, J., \& García, B. (2015). Validación de una escala de interacción en contextos virtuales de aprendizaje. Revista Electrónica de Investigación Educativa, 17(1), 116-129. http://redie.uabc.mx/vol17no1/contenido-berridi-etal.html

Britez, M. (2020). La educación ante el avance del COVID-19 en Paraguay. Comparativo con países de la Triple Frontera. SciELO - Scientific Electronic Library Online. https://doi.org/10.1590/SCIELOPREPRINTS.22

Carvalho, A., \& de Oliveira, K. (2016). Scale of strategies and motivation for learning in virtual environments. Revista Brasileira de Educacao, 21(66), 593-611. https://doi.org/10.1590/S1413-24782016216631

Castro-Rodríguez, Y., \& Lara-Verástegui, R. (2018). Perception of blended learning in the teaching-learning process by post-graduate students of Dentistry. Educacion Medica, 19(4), 223-228. https://doi.org/10.1016/j.edumed.2017.03.028 
Chakraborty, I., \& Maity, P. (2020). COVID-19 outbreak: Migration, effects on society, global environment and prevention. Science of the Total Environment, 728, 138882. https://doi.org/10.1016/j.scitotenv.2020.138882

Copari, F. (2014). La enseñanza virtual en el aprendizaje de los estudiantes del instituto superior tecnológico Pedro Vilcapaza - Perú. COMUNI@CCIÓN, 5(1), 14-21. http://www.scielo.org.pe/pdf/comunica/v5n1/a02v5n1.pdf

Córdova, R., Mamani-Benito, O., \& Tarqui, E. (2019). Estilos de aprendizaje y estrategias metacognitivas en estudiantes de psicología de una universidad privada de juliaca. Revista de Investigaciones de La Escuela de Posgrado, 8(3), 1224-1233. http://www.revistaepgunapuno.org/index.php/investigaciones/article/view/1574/26 9

Deshwal, P., Trivedi, A., \& Himanshi, H. (2017). Online Learning Experience Scale Validation and Its Impact on Learners' Satisfaction. Procedia - Computer Science, 112. https://doi.org/10.1016/j.procs.2017.08.178

Fernández, A., \& Rivero, M. (2014). Las plataformas de aprendizajes, una alternativa a tener en cuenta en el proceso de enseñanza aprendizaje. Revista Cubana de Informática Médica, 6(2), 207-221. http://scielo.sld.cu/pdf/rcim/v6n2/rcim09214.pdf

Furr, R. (2011). Scale Construction and Psychometrics for Social and Personality Psychology. https://doi.org/10.4135/9781446287866

Inzunza, B. C., Rocha, R. A., Márquez, C. G., \& Duk, M. S. (2012). Asignatura Virtual como Herramienta de Apoyo en la Enseñanza Universitaria de Ciencias Básicas: Implementación y Satisfacción de los Estudiantes. Formacion Universitaria, 5(4), 3-14. https://doi.org/10.4067/S0718-50062012000400002

Jelfs, A., \& Colbourn, C. (2002). Do Students' Approaches to Learning Affect their Perceptions of Using Computing and Information Technology? Journal of Educational Media, 27(1-2), 41-53. https://doi.org/10.1080/1358165020270104

João, M., \& Carvalho-Filho, M. (2020). Una nueva época para la educación médica después de la COVID-19. FEM: Revista de La Fundación Educación Médica, 23(2), 55-57. http://scielo.isciii.es/pdf/fem/v23n2/2014-9832-fem-23-2-55.pdf

Juca, F. X. (2016). La educación a distancia, una necesidad para la formación de los profesionales. Revista Universidad y Sociedad, 8(1), 106-111. http://scielo.sld.cu/pdf/rus/v8n1/rus15116.pdf

Lloret-Segura, S., Ferreres-Traver, A., Hernández-Baeza, A., \& Tomás-Marco, I. (2014). El análisis factorial exploratorio de los ítems: una guía práctica, revisada y actualizada. Anales De Psicología, 30(3), 1151-1169. https://www.redalyc.org/pdf/167/16731690031.pdf 
Mimirinis, M., \& Bhattacharya, M. (2007). Design of virtual learning environments for deep learning. Journal of Interactive Learning Research, 18(1), 55-64. https://www.learntechlib.org/p/21901/

Moneta, A., Montero, L., Juárez, M., Depetris, J., \& Fagnola, B. (2017). Adaptación y validación de un instrumento de medida para la interacción en b-learning. Virtualidad, Educación y Ciencia, 8(14), 27-41. https://revistas.unc.edu.ar/index.php/vesc/article/download/17330/17054

Moreira-Segura, C., \& Delgadillo-Espinoza, B. (2015). La virtualidad en los procesos educativos: reflexiones teóricas sobre su implementación. Revista Tecnología En Marcha, 28(1), 121. https://doi.org/10.18845/tm.v28i1.2196

Orozco, A. M., \& García, T. (2017). Autopercepción de habilidades de aprendizaje en ambientes virtuales. CPU-e. Revista de Investigación Educativa, 25, 144-167. http://www.scielo.org.mx/scielo.php?script=sci_arttext\&pid=S187053082017000200144\&lang=pt\%0Ahttp://www.scielo.org.mx/pdf/cpue/n25/18705308-cpue-25-00144.pdf

Palacios, M., Santos, E., Velásquez, M., \& León, M. (2020). COVID-19, una emergencia de salud pública mundial M. Revista Clínica Española, 1, 55-61 https://doi.org/10.1016/j.rce.2020.03.001

Prieto, G., \& Delgado, A. R. (2010). Fiabilidad y validez. Papeles Del Psicologo, 31(1), 67-74. http://www.papelesdelpsicologo.es/pdf/1797.pdf

Saavedra-Idrogo, F., \& Saavedra-Paredes, L. (2017). Entorno virtual Moodle en la mejora del aprendizaje de cirugía en estudiantes de medicina de la Universidad Católica Santo Toribio de Mogrovejo. Revista de La Fundación Educación Médica, 20(5), 241. https://doi.org/10.33588/fem.205.908

Shereen, M. A., Khan, S., Kazmi, A., Bashir, N., \& Siddique, R. (2020). COVID-19 infection: Origin, transmission, and characteristics of human coronaviruses. Journal of Advanced Research, 24, 91-98. https://doi.org/10.1016/j.jare.2020.03.005

Taber, K. S. (2018). The Use of Cronbach's Alpha When Developing and Reporting Research Instruments in Science Education. Research in Science Education, 48(6), 1273-1296. https://doi.org/10.1007/s11165-016-9602-2

UNESCO. (2020). Propuestas de la UNESCO para garantizar la educación online durante la pandemia. https://www.educaweb.com/noticia/2020/04/01/propuestasunesco-garantizar-educacion-online-pandemia-19132/.

Ventura-León, J. (2019). De regreso a la validez basada en el contenido. Adicciones, O(0). https://doi.org/10.1080/00221300309601160.Cohen

Viner, R. M., Russell, S. J., Croker, H., Packer, J., Ward, J., Stansfield, C., Mytton, O., Bonell, C., \& Booy, R. (2020). School closure and management practices during coronavirus outbreaks including COVID-19: a rapid systematic review. The Lancet Child and Adolescent Health, 4(5), 397-404. https://doi.org/10.1016/S2352- 
Diseño y validación de una escala de uso de medios académicos virtuales durante la pandemia COVID-19

4642(20)30095-X 